\title{
A Novel Interface for High-Sensitive Immunoassay Using Orientation Controlled Protein A and Non-biofouling Phospholipid Polymer Surface
}

\author{
Nobuyuki Tajima ${ }^{1}$, Ryosuke Matsuno ${ }^{1,3}$, Tomohiro Konno ${ }^{1,3}$, Madoka Takai ${ }^{1,3}$, Kazuhiko \\ Ishihara ${ }^{1,2,3}$ \\ ${ }^{1}$ Department of Materials Engineering, ${ }^{2}$ Department of Bioengineering, ${ }^{3}$ Center for NanoBio Integration (CNBI), \\ The University of Tokyo, 7-3-1 Hongo, Bunkyo, Tokyo, Japan. FAX +81-3-5841-8647. \\ Email: ishihara@mpc.t.u-tokyo.ac.jp
}

\begin{abstract}
A novel procedure for immobilizing orientation-controlled antibodies was investigated using tyrosinase catalyzed immobilization of Staphylococcal protein A (SpA). We synthesized welldefined structured block-type polymer, poly(2-methacryloyloxyethyl phosphorylcholine(MPC)-block-2-aminoethyl methacrylate (AEMA)) (PMbA) from the substrate by surface-initiated living radical polymerization for bioconjugation. One of the segments in the PMbA, poly(MPC) provides hydrophilicity and biocompatibility and the other segment, poly(AEMA) possesses amino groups for conjugation with biomolecules. On this $\mathrm{PMbA}$ polymer grafted substrates, $\mathrm{SpA}$ is first oxidized by tyrosinase and then immobilized to the amino group of the substrate through the active quinone groups in the SpA. The PMbA substrates showed a good resistance for non-specific protein adsorption, due to its primal layer of poly(MPC) segments. As the result of sandwich immunoassay, this system using tyrosinase and SpA showed the best primary antibody/secondary antibody ratio among the system without $\mathrm{SpA}$ and that with randomly oriented $\mathrm{SpA}$. This procedure can be further utilized for achieving higher sensitivity of immunoassay systems.
\end{abstract}

Keyword: MPC polymer, biointerface, immunoassay, surface-initiated living radical polymerization, Protein A.

\section{INTRODUCTION}

Because of their high selectivity, biomolecules such as antibodies and enzymes have been used for biosensing devices. Their innate high selectivity, however, must be sacrificed in preparation of biosensors; the denaturation of the surface-bound biomolecules[1] and the sterically hindered active sites of immobilized proteins[2-4] are the two major drawbacks upon immobilization of these bio-functional proteins. Particularly in immunosensors, in which a high selectivity is achieved with immobilized antibodies, the controlled orientation of surface-bound antibodies greatly affects the sensitivity of the sensing system[3]. Staphylococcal Protein A (SpA) is one of the known ligands that specifically bind biomolecules with highly oriented and organized maner; in this case, SpA bindis a $\mathrm{Fc}$ region of $\mathrm{IgG}$ antibody. Ahmed et al. reported tyrosinase catalyzed $\mathrm{SpA}-\mathrm{NH}_{2}$ binding chemistry conserved the SpA's IgG binding site on the other side of amine-functionalized surface[5]. In addition to the loss of biomolecules' natural selectivity, any proteins can be non-specifically adsorbed to the substrates,

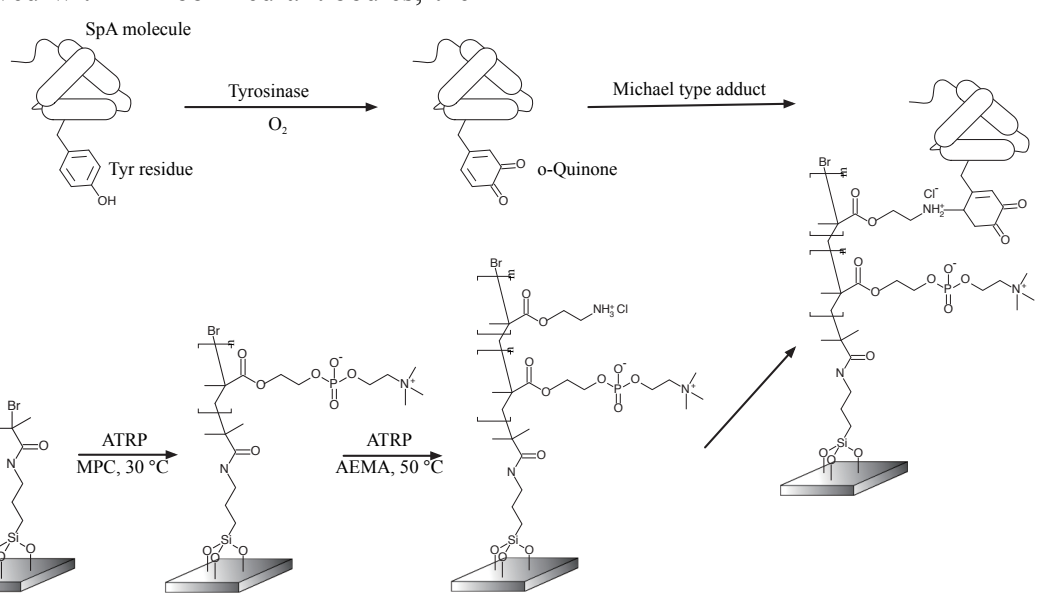

Figure 1. Schematic representation of preparation procedure of poly(MPC-block-AEMA) grafted surface and conjugation of SpA 
decreasing the sensitivity of these systems. Many surface modification techniques have been invented to prevent the non-specific protein adsorption[6-8]. Among these approaches, 2-methacryloyloxyethyl phosphorylcholine (MPC) polymer exhibits a good resistance to non-specific interactions to plasma proteins[8]. In this report we present a novel interface for a high-sensitive immunoassay with poly(MPCblock-2-aminoethyl methacrylate (AEMA) (PMbA) grafted surface, on top of which is immobilized SpA via tyrosinase chemistry (Figure 1). In this research, the combination of non-biofouling MPC polymer and the orientation controlled $\mathrm{SpA}$ was achieved and evaluated.

\section{EXPERIMENTAL SECTION}

\subsection{Materials}

MPC was synthesized using a reported method and purified by recrystalization from acetonitrile[9]. AEMA were purchased from Polysciences inc. (Pennsylvania, USA), respectively. Fluorescence labeled and nonlabeled goat anti-human CRP antibodies were purchased from Rockland Immunochemicals (Pennsylvania, USA) and antigens, C-Reactive Protein (CRP), was purchased from Oriental Yeast Co. (Tokyo, Japan). SpA was purchased from MP Biochemicals (California, USA). All other organic reagets were purchased from Wako Pure Chemicals (Tokyo, Japan) and used with no further purification.

2.2 Preparation of atom transfer radical polymerization (ATRP) initiator immobilized surface

The surface initiator, 3-(2-bromoisobutyramido) propyl(trimethoxy)silane (BAPTMS), were synthesized as reported[10]. Silicon substrates $\left(\mathrm{SiO}_{2} / \mathrm{Si}\right)$ were cleansed using oxygen plasma for 5 mins at $300 \mathrm{~W}$. The cleansed substrates were dipped in ethanol solution containing $0.05 \mathrm{wt} \%$ of deinonized water, $1 \mathrm{wt} \%$ of BAPTMS and $0.01 \mathrm{wt} \%$ of succinic acid for 3 hours, followed by heating at $110{ }^{\circ} \mathrm{C}$ for 1 hour to form covalent bonding of initiator and silicon substrates

\subsection{Surface-initiated ATRP}

To a flask containing MPC (2078 $\mathrm{mg}, 0.7000$ $\mathrm{mmol}), \mathrm{Cu}(\mathrm{I}) \mathrm{Br}(18.8 \mathrm{mg}, 0.0140 \mathrm{mmol})$ and 2,2'bipyridyl (44.0 $\mathrm{mg}, 0.0280 \mathrm{mmol})$, thoroughly degassed methanol $(10 \mathrm{~mL})$ was poured under Ar gas flow. After the reagents were completely dissolved, 2 bromoisobutyryl bromide $(21.6 \mu \mathrm{L}, 0.0140 \mathrm{mmol})$ and initiator immobilized silicon substrates were added to the reaction flask. The polymerization was taken place under $30{ }^{\circ} \mathrm{C}$ for 20 hours. Similar procedures were conducted for AEMA polymer brush; AEMA (1166 mg, $0.7000 \mathrm{mmol})$ instead of MPC, $\mathrm{Cu}(\mathrm{I}) \mathrm{Br}(188 \mathrm{mg}, 0.140$ mmol), 2,2'-bipyridyl (440 $\mathrm{mg}, 0.280 \mathrm{mmol}$ ), 2bromoisobutyryl bromide $(216 \mu \mathrm{L}, 0.140 \mathrm{mmol})$ and methanol:water $(8: 2)$ mixed solution were used. The polymerization of AEMA was conducted at $50{ }^{\circ} \mathrm{C}$ for more than 6 hours. The target degree of polymerization for poly(MPC) was set to 50 and poly(AEMA) and $\mathrm{PMbA}$ to 10 , unless otherwise noted. A small potion of the reaction solution was taken and monitored using ${ }^{1} \mathrm{H}-$ NMR (JEOL JNM-NR30, Tokyo, Japan) and GPC (OHpak SB-803 HQ column, Shodex, Tokyo, Japan) measurements to follow the polymerization kinetics of poly(MPC) and poly(AEMA)

2.4 Surface characterization of polymer grafted surfaces
Surface characterization of polymer grafted substrates were carried out using X-ray photoelectron spectroscopy (XPS: AXIS-His; Shimadzu/Kratos, Kyoto, Japan) and ellipsometry measurements. The binding energy of N 1s and P 2p orbitals was closely monitored with XPS. Ellipsometric thickness was calculated using monochromatic light and fitted using $\mathrm{n}=1.48$ and $\mathrm{k}=0$ as previously reported[11] (DVA-36L3; Mizojiri Optical Co. Ltd., Tokyo Japan. light-source: He-Ne laser $632.8 \mathrm{~nm}$ ).

2.5 Non-specific protein adsorption on polymer grafted surfaces

Poly(MPC), poly(AEMA) and PMbA grafted $\mathrm{SiO}_{2} /$ $\mathrm{Si}$ substrates were prepared. These substrates were immersed in $10 \mathrm{mg} / \mathrm{mL}$ PBS solution of bovine serum albumin (BSA) $(1: 4=$ fluorescein-conjugated BSA (FITC-BSA) : non-labeled BSA) for 1.5 hours. After through rinsing, the fluorescence was measured using the fluorescent scanner (Typhoon 9410; Amersham Biosciences UK Ltd., Buckinghamshire, UK).

2.6 Time-lapse UV observation

A PBS solution of tyrosinase (120 units $/ \mathrm{mL}$ ), SpA $(100 \mu \mathrm{g} / \mathrm{mL})$ and the mixture of tyrosinase and SpA were prepared and used for time-lapse UV observation at $280 \mathrm{~nm}$ and $475 \mathrm{~nm}$. Before and during the measurements, the solutions were placed under oxygen atmosphere.

2.7 Optimization of tyrosinase catalyzed SpA immobilization

The optimum conditions of tyrosinase catalyzed $\mathrm{SpA}$ immobilization were investigated. In the first set, the amount of tyrosinase units were varied from 0 to 250 units $/ \mathrm{mL}$ while the amount of $\mathrm{SpA}$ was kept constant at $50 \mu \mathrm{g} / \mathrm{mL}$. Then the reaction time was controlled from 0 to 60 mins. The reaction time is the time from mixing tyrosinase to $\mathrm{SpA}$ to exposing this mixture to $\mathrm{PMbA}$ substrates.

2.8 Sandwich assay and evaluation of antibody orientation

PMbA grafted substrates were used to prepare three differently modified interfaces to immobilize primary antibodies: direct immobilization, $\mathrm{SpA}$ mediated immobilization and orientation-controlled SpA mediated immobilization. The direct immobilization of primary antibody was achieved by exposing the $\mathrm{SpA}$ solution $(25$ $\mu \mathrm{g} / \mathrm{mL}$ ) to the substrate for 6 hours. The SpA mediated and the orientation-controlled SpA mediated substrates were prepared by exposing SpA only and $\mathrm{SpA}$ and tyrosinase solutions to the $\mathrm{PMbA}$ substrates prior to immobilizing primary antibodies. In this assay, $50 \mu \mathrm{g} /$ $\mathrm{mL}$ PBS solution of non-labeled antibody was used for both primary antibody conjugation. The FITC-labeled antibody was used instead, upon quantifying the amount of the primary antibodies and the secondary antibodies. The antigen, human CRP, was used with the concentration of $50 \mu \mathrm{g} / \mathrm{mL}$. The amount of primary and secondary antibodies was quantified using FITCconjugated antibody and fluorescent scanner.

\section{RESULTS AND DISCUSSIONS}

3.1 The kinetics of living polymerization

The living polymerization was confirmed from the linearity of $k_{p}$ against the polymerization time and of the molecular weight against the conversion (data not 
shown). The polydispersity is as low as 1.16 , which also supports the living polymerization of poly(MPC). Similar results were also obtained for poly(AEMA).

\subsection{Surface characterization}

XPS and ellipsometry were carried out to characterize polymer grafted surfaces. The XPS spectra of unmodified $\mathrm{SiO}_{2} / \mathrm{Si}$ surface, poly(MPC) grafted surface and $\mathrm{PMbA}$ grafted surface are shown in Figure 2. poly(MPC) grafted substrate showed unique peak at $134 \mathrm{eV}$ and $403 \mathrm{eV}$, attributable to the phosphoryl group and the quaternary ammonium salt group of MPC. PMbA substrate showed an additional peak at $399 \mathrm{eV}$ which can be assigned to its primary amine group. The ellipsometry measurement was conducted and gave the thickness of poly(MPC) and PMbA grafted surface as $8.3 \pm 0.8 \mathrm{~nm}$ and $10.4 \pm 0.4 \mathrm{~nm}$, respectively. The graft density of the poly(MPC) brush was calculated as 0.42 chains $/ \mathrm{nm}^{2}$ with the following formula;

$$
\sigma=\frac{\rho h N_{\mathrm{A}}}{M}
$$

where $\sigma$ is the graft density, $\rho$ is the density of the polymer (as $1.3 \mathrm{~g} / \mathrm{cm}^{3}$ for MPC polymer[11]), $N_{\mathrm{A}}$ is the Avogadro's number and $M$ is the molecular weight of the polymer. With these results, the successful surfaceinitiated polymerization of poly(MPC) and PMbA was confirmed.
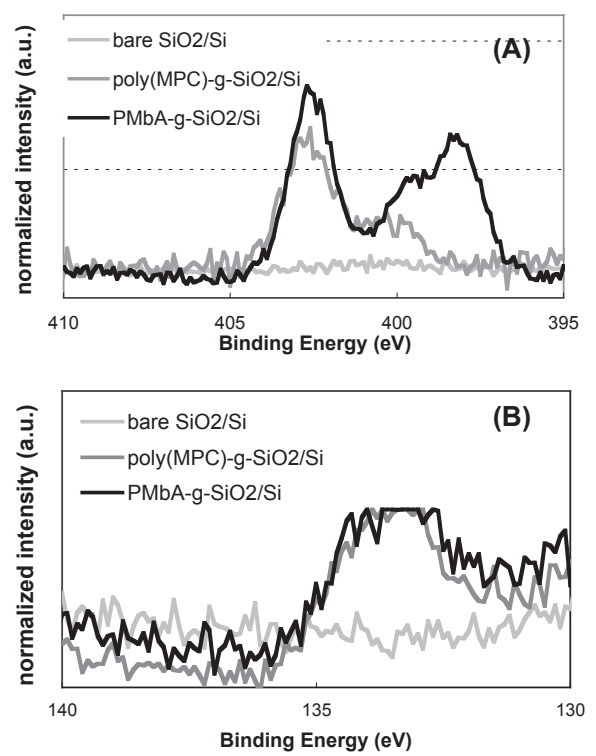

Figure 2. XPS spectra of (A) $\mathrm{N} 1 \mathrm{~s}$ and (B) $\mathrm{P} 2 \mathrm{p}$ for poly(MPC), PMbA grafted and unmodified substrate.

3.3 Non-specific protein adsorption on polymer grafted surface

After immersed in BSA/FITC-BSA solution for 1.5 hours, the polymer grafted substrates were processed using the fluorescence scanner. The amount of adsorbed BSA is shown in Figure 3. On the poly(MPC) grafted substrates, no adsorption of BSA was observed as expected. Both on PMbA and poly(AEMA) substrates, the amount of adsorbed BSA increased along with the AEMA units. The amount of adsorbed BSA on PMbA substrates was decreased to about one third of that on poly(AEMA) substrates, even though the top most layer of AEMA chains were set to be the same. This decrease of non-specifically bound BSA on PMbA substrates can

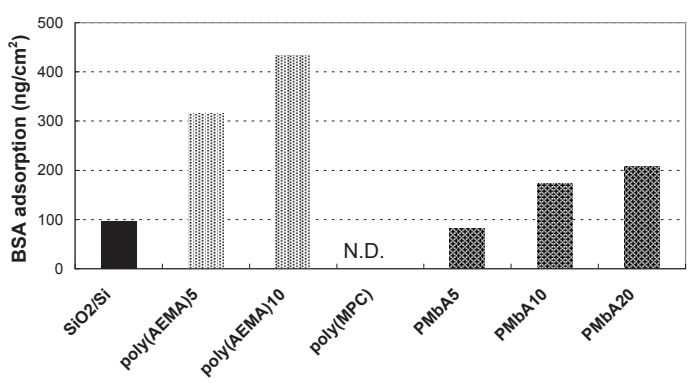

Figure 3. Non-specific BSA adsorption on surfaces. The number after the abbreviation corresponds the number of AEMA units in poly(AEMA) and PMbA polymer chains (in feed). N.D.= not detected.

be considered as the result of primal poly(MPC) layer exhibiting its unique features. The non-specific protein adsorption on poly(MPC) grafted substrates and the graft density of the polymer is thoroughly studied by Feng et al.[12,13]. According to their results, the poly(MPC) grafted substrates with the graft density of 0.39 chains $/ \mathrm{nm}^{2}$ exhibits almost no adsorption of fibrinogen on the substrate, even from the chain length of 10 units of MPC. Our substrate showed a similar graft density and hence we think it is sufficient for the poly(MPC) layer in PMbA to exhibit its property. Also the shown values are the average of the two independent experiments and their tendencies are thought to be reliable.

\subsection{Time-lapse UV observation}

To confirm the oxidation of SpA's tyrosine residue by tyrosinase, time-lapse UV measurements were conducted. The observed wavelengths were $280 \mathrm{~nm}$ and $375 \mathrm{~nm}$, representing the absorbant wavelengths of dihydroxy-phenylalanine (DOPA), an intermediate product, and that of DOPA-quinone, the final oxidized product, respectively. The resultant UV spectra are shown in Figure 4. Although the solutions containing SpA only and tyrosinase gave the steady UV absorbance at both wavelengths, the mixed solution of these two gave a rise along with the time. That is to say, the tyrosinase does oxidize the tyrosine residue of $\mathrm{SpA}$ into the $o$-quinone form.
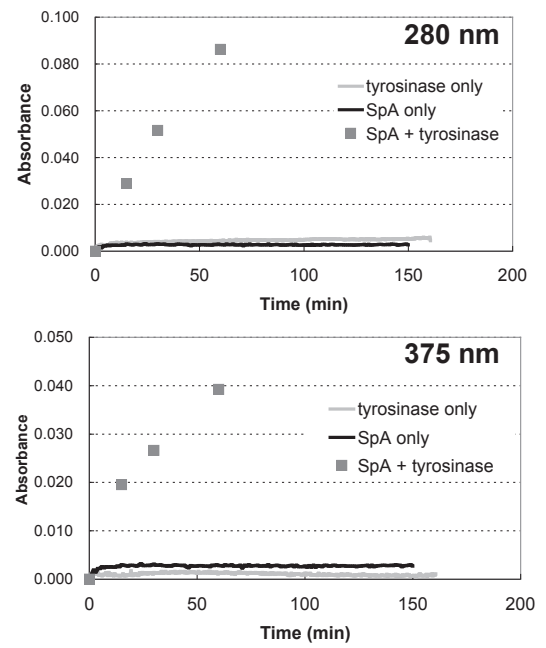

Figure 4. Time-lapse UV spectra of SpA only, tyrosinase only and the mixed solution of SpA and tyrosinase at $280 \mathrm{~nm}$ (top) and $375 \mathrm{~nm}$ (bottom) 

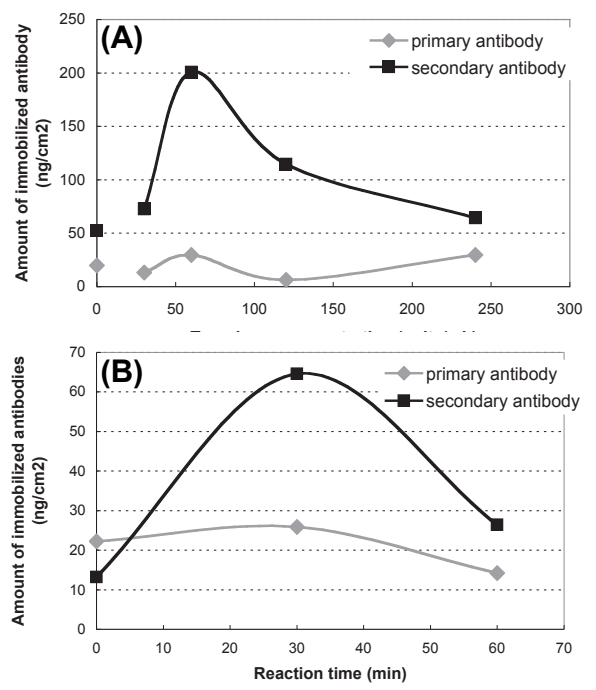

Figure 5. Effects of various conditions of tyrosinase catalyzed $\mathrm{SpA}$ immobilization method on amount of immobilized antibody. (A): tyrosinase amount variant. (B): the waiting time variant.

\subsection{Optimization of tyrosinase catalyzed SpA immobilization}

In order to optimize the tyrosinase catalyzed SpA immobilization method, a sandwich immunoassay was conducted with various conditions. The special attention was paid to two variants: the ratio of tyrosinase to $\mathrm{SpA}$ and the reaction time between mixing $\operatorname{SpA}$ and tyrosinase and exposing the mixture to the substrates. Figure 5 shows the amount of immobilized antibodies with different tyrosinase units (5-A) and with the different reaction time (5-B). Both Fig.s proves the distinct optimum values exist in these two variables. With these optimum conditions, the rest of sandwich immunoassays were conducted.

3.7 Sandwich immunoassay and evaluation of antibody orientation

The effect of orientation-controlled immobilization of antibody was assessed using the sandwich immunoassay (Figure 6). The amount of immobilized primary antibodies on non-SpA assisted interface is as high as $70 \mathrm{ng} / \mathrm{cm}^{2}$ and the amount of secondary antibody was about $100 \mathrm{ng} / \mathrm{cm}^{2}$ with the ratio of the secondary/ primary ratio being about 1.6. On the SpA mediated interface, while the amount of immobilized primary antibodies was increased to $85 \mathrm{ng} / \mathrm{cm}^{2}$, the ratio of the secondary to the primary antibody was about the same level (1.7) compared to the non-SpA assisted system. Although $\mathrm{SpA}$ is well-known for its selectivity to $\mathrm{Fc}$ region of antibodies, the expected increase in the sensitivity was not fully observed in these sets of experiments using physically and randomly captured SpA molecules on PMbA substrates. By using tyrosinase catalyzed immobilization of SpA, however, the ratio of the secondary antibody to the primary antibody was greatly improved up to 8.1 . These results suggest that the tyrosinase catalyzed immobilization of $\mathrm{SpA}$ is a good way to immobilize antibodies in a highly oriented manner.

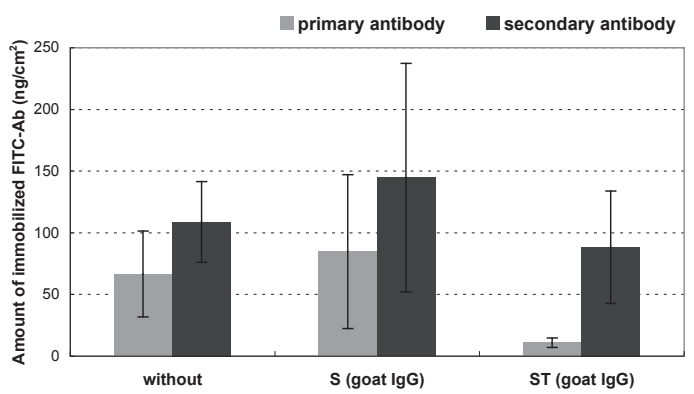

Figure 6. Effect of orientation-controlled immobilization. Antibodies were immobilized on the substrate directly (without $\mathrm{SpA}$ ), through randomlyoriented $\mathrm{SpA}$ (SpA only) and through orientation controlled $\mathrm{SpA}(\mathrm{SpA}+$ tyrosinase $)$

\section{CONCLUSIONS}

A novel interface was prepared and evaluated, in which the orientation controlled antibodies were immobilized to the oriented SpA molecules on top of the biocompatible MPC polymer. The controlled orientation of $\mathrm{SpA}$ was achieved using the tyrosinase catalyzed immobilization technique. This interface not only showed good resistance for non-specific protein adsorption, but exhibited the good secondary-to-primary antibody ratio that suggests controlled orientation of immobilized antibodies.

\section{REFERENCES}

[1] J. E. Butler, L. Ni, W. R. Brown, K. S. Joshi, J. Chang, B. Rosenberg and E. W. J. Voss, Mol. Immunol., 30, 1165 (1993).

[2] S. Chen, L. Liu, J. Zhou and S. Jiang, Langmuir, 19, 2859, (2003).

[3] R. Danczyk, B. Krieder, A. North, T. Webster, H. HogenEsch and A. Rundell, Biotechnol. Bioeng., 84, 215, (2003).

[4] Y. Jung, J. Y. Jeong and B. H. Chung, The Analyst, 133, 697 (2008).

[5] S. R. Ahmed, A. T. Lutes and T. A. Barbari, J. Membr. Sci., 282, 312 (2006).

[6] K. Ishihara, H. Nomura, T. Mihara, K. Kurita, Y. Iwasaki and N. Nakabayashi, J. Biomed. Mater. Res., 39, 323, (1998).

[7] S. Sakaki, N. Nakabayashi and K. Ishihara, J. Biomed. Mater. Res., 47, 523, (1999).

[8] Y. Iwasaki, S. Sawada, N. Nakabayashi, G. Khang, H.B. Lee, K. Ishihara, Biomaterials, 20, 2185, (1999).

[9] K. Ishihara, T. Ueda, N. Nakabayashi, Polym. J., 22, 355, (1990).

[10] S. Tugulu, A. Arnold, I. Sielaff, K. Johnsson and HA. Klok, Biomacromolecules, 6, 1602 (2005).

[11] R. Iwata, P. Suk-In, V. P. Hoven, A. Takahara, K. Akiyoshi and Y. Iwasaki, Biomacromolecules, 5, 2308, (2004).

[12] W. Feng, J. Brash and S. Zhu, Biomaterials, 27, 847 (2006).

[13] W. Feng, S. Zhu, K. Ishihara and J. Brash, Biointerphases, 1, 50 (2006).

(Received January 4, 2009; Accepted February 24, 2009) 